\title{
Wavelet-based approach to evaluation of signal integnity
}

Jan T. Bialasiewicz, Senior Member, IEEE, David González, Member, IEEE, Josep Balcells, Senior Member, IEEE , and Javier Gago

\begin{abstract}
In this paper, we present a new approach to evaluation of signal integrity that is based on signal energy density as a function of time and frequency, represented by its wavelet scalogram. Using signal integrity ratio and cumulative energy ratio, we illustrate signal integrity analysis with simulated examples, followed by the demonstration of their usefulness through analysis of experimental data of a real audio amplifier. These figures of merit represent the extent to which the integrity of a signal is diminished by the electromagnetic interference effects and/or nonlinear processes.
\end{abstract}

IndexTerms: Electromagnetic interference (EMI) effects, signal integrity, signal processing with wavelets, wavelet scalogram.

\section{INTRODUCTION}

In recent years, we can notice a substantial growth of research that aims to detect, evaluate, and reduce electromagnetic interferences (EMIs) induced in electronic equipment. This trend is due to the increasing interest in improving the performance quality, reliability, and security of such equipment.

One of the common methods of evaluation of signal characteristics is based on signal analysis in time domain. Other useful representations involve the frequency-domain view of a signal and are based on the Fourier transform. Although frequency-domain representations, such as the power spectrum of a signal, provide useful information, they do not show how the frequency content of a signal evolves over time. This task has to be approached by simultaneous analysis in the time and frequency domains.

To analyze the low frequency content, we need to look at signals over longer time intervals, whereas the time-varying high frequency content has to be evaluated over shorter time intervals. This is the feature of the wavelet transform (WT) that provides high time resolution for the high frequency range and low time resolution for the low frequency range.

Multiresolution and wavelet theory have been recently applied to analyze electromagnetic compatibility (EMC) issues. A wavelet-based analysis tool for 
EMI noise feature extraction, intended for identification of the noise source nature, appears in [1]. Continuous WT (CWT) can also be used to evaluate the effectiveness of the EMI reduction techniques applied to switched power converters [2]. On the other hand, wavelet concepts can be used to speed up the solution of some complex mathematical EMC problems by reducing the volume of computational requirements, as shown in [3] in the case of analysis of lightning effects induced on multiconductor transmission lines. Diagnosis of electronic hardware, such as a printed circuit board, is also within the scope of wavelet applications. In [4], line termination faults are identified by means of the wavelet-based analysis of the crosstalk phenomena.

Similar applications have also been proposed in other remarkable disciplines such as data compression, signal processing, image analysis, system control, fault detection, statistics, and modeling of nonlinear dynamic processes [5], [6], [7], [8], [9], [10], [11], [12], [13], [14], [15], [16], [17]. A useful overview of the subject can be found in [18] and [19].

In this research, we have developed a general procedure along with new tools for evaluation of influence of the EMI and system nonlinearities on system performance as evaluated by signal integrity. For the first time, we have given the original formulation of this concept in [20], and with limited success, we attempted its application to the power quality evaluation. We propose to evaluate signal integrity using time-frequency representations of the energy density of signals. In particular, using the wavelet scalogram, we partition the time-frequency plane into frequency subbands used to represent the time evolution of frequency components of signal energy density. Implementing this concept, we are able to determine a time-frequency domain (which includes one or more frequency subbands), in which most, for example, 95\%, of the energy of an input signal of a system is contained. As a result of nonlinearities and/or the EMI, substantial energy of the output signal may appear outside this frequency domain. We have introduced performance indexes related to this concept. However, it is a general-purpose methodology, and the evaluation of the EMI effect on system performance, as represented by signal integrity, is only one of its possible applications.

We illustrate our methodology by showing its application to integrity evaluation of a signal that passes through an audio amplifier. This integrity may be compromised by the EMI, as well as inherent nonlinearities of an amplifier. It is natural to assume that the signal integrity is preserved if the frequency content of the amplifier output signal as a function of time is identical to that of the input signal. Since the gain of an audio amplifier should be constant in time and frequency within a required amplifier bandwidth, the introduced concept of signal integrity may, most conveniently, be represented in terms of local energy densities of an ideal output signal and an actual output signal. The energy density of signals as a function of frequency can be represented by their wavelet scalograms that are defined using a CWT.

The problems of the wavelet applications to EMC are discussed in [21], [22], [23]. An interesting approach to identification of the EMI noise sources in power converters, using both discrete and continuous-time WTs, is presented in [24] 
but could be substantially enriched by the application of time-frequency energy density representation introduced in this paper. The effectiveness of the wavelet representation of audio transients, as well as their application to EMC/EMI system performance analysis, is evidenced in [25] and [26].

In Section II, we give a brief presentation of the relationship between scale and frequency, followed by an introduction to scalograms, and presentation of the proposed tools and simulated examples of signal integrity analysis. Their application to signal integrity analysis, using experimental data of a real audio amplifier, is presented in Section III, and Section IV summarizes this paper.

\section{WT, SIGNAL ENERGY, AND SIGNAL INTEGRITY REPRESENTATION}

In this section, we first introduce the CWT with emphasis on the relationship between scale and frequency. This relationship is of fundamental importance to the scalogram representation of the local energy density of signals and, consequently, to the evaluation of the integrity of signals that may be corrupted by the EMI.

The CWT $W f(s, b)$ of a finite-energy signal $f(t)$ is defined as its scalar product with the wavelet $\psi_{s b}(t)$ as follows:

$$
W f(s, b)=\int_{-\infty}^{\infty} \psi_{s b}(t) f(t) d t=\frac{1}{2 \pi} \int_{-\infty}^{\infty} \hat{f}(\omega) \hat{\psi}^{*}(s \omega) e^{j b \omega} d \omega
$$

with

$$
\psi_{s b}(t)=s^{-\frac{1}{2}} \psi\left(s^{-1}(t-b)\right), \quad s \in R^{+} ; \quad b \in R
$$

where $\psi(t)$ is a mother wavelet. Varying $b$ for the fixed scale $s$, we are sliding a wavelet with a fixed bandwidth $B_{s}$ and with some fixed center frequency $\omega_{s}$ along the analyzed signal.

We need to establish the relationship between the scale sand the frequency content, at this scale, of a particular analyzing wavelet. This is accomplished by defining two frequency characteristics of the mother wavelet [18]: the center frequency

$$
\omega_{0}=\frac{1}{2 \pi} \int_{0}^{\infty} \omega|\hat{\psi}(\omega)|^{2} d \omega
$$


and the bandwidth

$B_{0}=\sigma_{\omega}$, centered around $\omega_{0}$, with

$\sigma_{\omega}^{2}=\frac{1}{2 \pi} \int_{0}^{\infty}\left(\omega-\omega_{0}\right)^{2}|\hat{\psi}(\omega)|^{2} d \omega$.

For a wavelet of the scale $s$, the center frequency and the bandwidth are respectively defined by the following equations:

$\omega_{s}=\frac{\omega_{0}}{s} \quad B_{s}=\frac{B_{0}}{s}=\frac{\omega_{\max }-\omega_{\min }}{s}$

with $\omega_{\max }=\omega_{0}+B_{0} / 2$ and $\omega_{\min }=\omega_{0}-B_{0} / 2$. Note that, by this convention, $B_{0}=B_{1}$ and $\omega_{0}=\omega_{1}$.

It is important to choose the mother wavelet with high frequency resolution or narrow bandwidth. The increase of the scale simproves the frequency resolution at lower frequencies. This is illustrated in Fig. 1, showing the magnitude of the Fourier transform of the Morlet wavelet for several scales $s(s=1,2,3,4,5)$. The Morlet mother wavelet is given by the following equation (used as justified in the Appendix):

$\psi(t)=e^{\frac{-t^{2}}{2}} \cos (5 t)$.

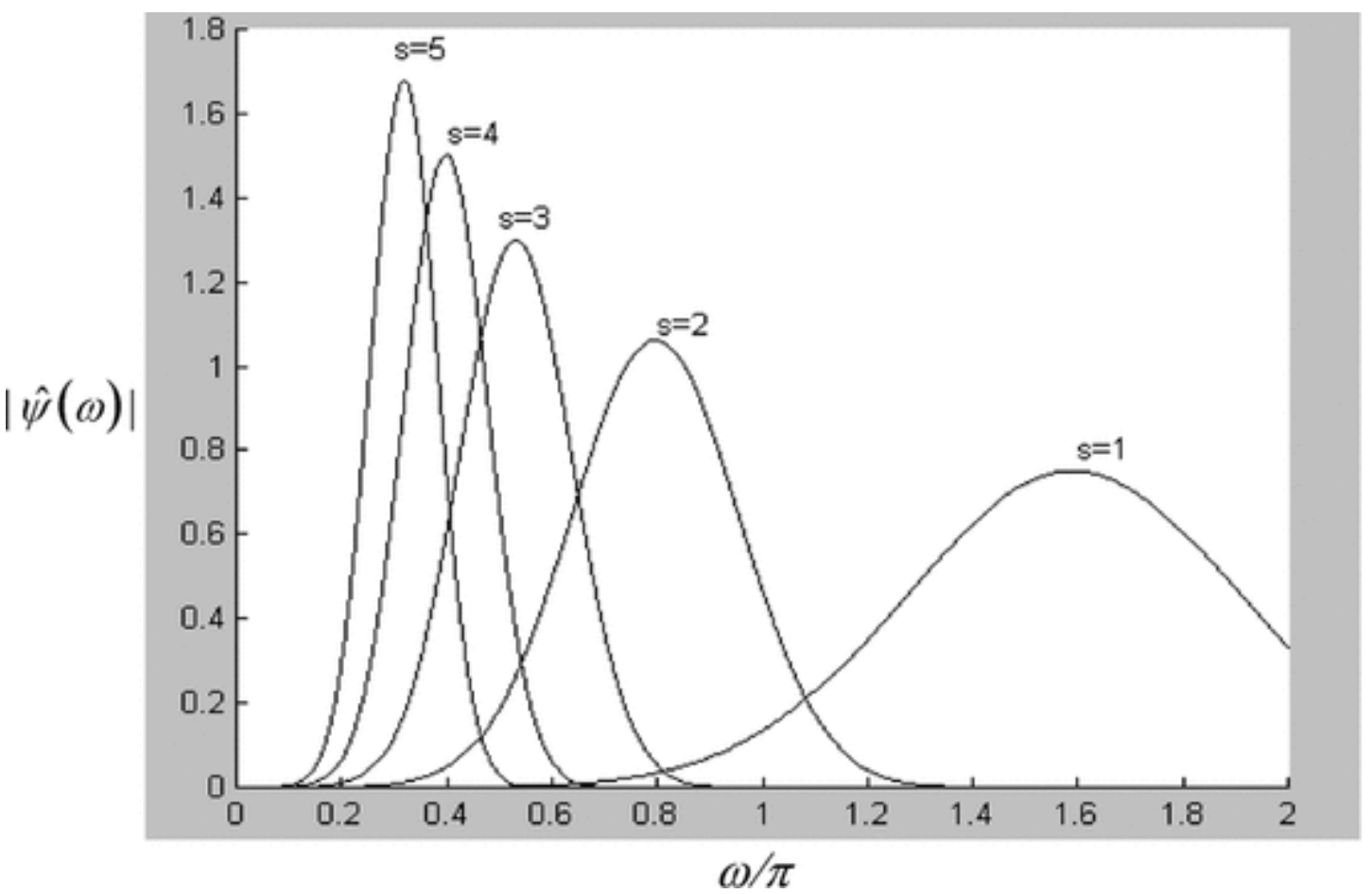

Fig. 1 Fourier transform of Morlet wavelet for different scales. 

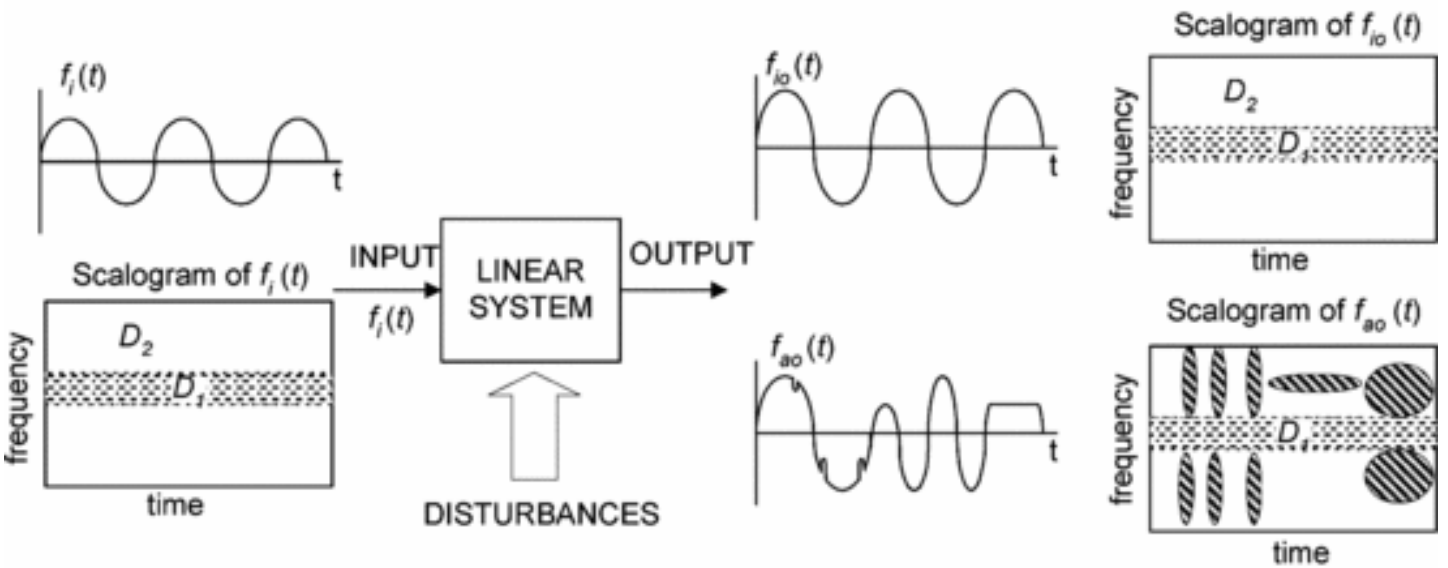

ACTUAL OUTPUT

Fig. 2. Signal energy density in the time-frequency domain illustrates the output signal distortion of a linear system.

For the Morlet wavelet, $\omega_{0}=5$, and $B_{0}=1.701$ (radian units). Using these two parameters, we can determine $\omega_{\text {sand }} B_{s}$ for any value of $s$. To convert frequency wradians to radians per second, we have to multiply the ratio $\omega / \pi$ by the Nyquist frequency $(1 / 2) \omega_{T} \mathrm{rad} / \mathrm{s}$. For example, setting $s=5$, we obtain the center frequency $\omega_{5}=\left(\left(\omega_{0} / 5\right) / \pi\right)\left((1 / 2) \omega_{T}\right)=(1 / 2 \pi) \omega_{T}$ and the bandwidth $B_{5}=\left(\left(B_{0} / 5\right) / \pi\right)\left((1 / 2) \omega_{T}\right)=(0.3402 / 2 \pi) \omega_{T} \mathrm{rad} / \mathrm{s}$. In other words, for a constant scale, we can vary the frequency range of analysis using different sampling frequencies $\omega_{T}$. It is clear from Fig. 1 that the magnitude of the Fourier transform of the Morlet wavelet for $s=1$ is almost entirely positioned to the right of $\omega / \pi=1$ or to the right of the Nyquist frequency. In other words, it is almost completely uncorrelated with the discrete representation of the original signal, or the useful bandwidth of the Morlet mother wavelet is almost entirely above half of the sampling frequency. It is apparent from this figure that the frequency resolution is much better for the lower relative frequencies (or for the higher scales). This immediately offers a solution to the problem of poor resolution at higher frequencies, i.e., the analyzed signal can be oversampled at such a rate that lower scales and, in particular, $s=1$ will not contain any useful frequencies. In other words, the bands of the lower scale wavelets (or wavelets with frequency resolutions too small for the performed analysis) will be outside the essential bandwidth of the analyzed signal.

Representing wavelet atoms $\psi_{b s}(t)$ in the frequency domain, we can say that most of their energy is in the frequency interval of the length of $B_{s}=B_{0} / \mathrm{s}$ centered around $\omega_{s}=\omega_{0} / \mathrm{s}$ or in the time domain around $b \in R$ in the interval of the length $s \sigma_{t}$ with the standard deviation $\sigma_{t}$ defined by the following integral: $\sigma_{t}^{2}=\int_{-\infty}^{\infty} t^{2}|\psi(t)| d t$ 
The standard deviation of the Morlet wavelet $\sigma_{t}=1.5944$. Note that, in the timefrequency plain, we have characterized a mother wavelet by three parameters: $\omega_{0}, \sigma_{\omega}\left(\right.$ or $\left.B_{0}\right)$, and $\sigma_{t}$. This defines, for every wavelet $\psi_{b s}(t)$, the Heisenberg box [18] in the time-frequency plane, centered at $\left(b, \omega=\omega_{0} / s\right)$ with the dimension of $s \sigma_{t}$ along the time axis and $\sigma_{\omega} / s_{\text {along }}$ the frequency axis. Its area $\sigma_{t} \sigma_{\omega}$ is independent of frequency or scale, and its sides represent local time and frequency resolution. For a fixed scale (or frequency), the dimension of the Heisenberg boxes in time direction is constant but (for a fixed time) decreases as frequency increases or is proportional to scale.

The scalogram of a signal $f(t)$, denoted by $P_{W} f\left(b, \omega_{s}\right)$, is defined as $P_{W} f\left(b, \omega_{s}\right)=|W f(b, s)|^{2}=\left|W f\left(b, \frac{\omega_{0}}{s}\right)\right|^{2}$.

The magnitude square of a wavelet coefficient in (8) represents a local timefrequency energy density, which measures the energy of $f$ in the Heisenberg box of each wavelet $\psi_{b s}(t)$ centered at $\left(b, \omega=\omega_{0} / s\right)$. This description of signal energy (in the timescale domain) facilitates the identification of time-varying energy flux, spectral evolution, and transient bursts not readily visible in time- or frequency-domain representations [6].

We will show now that the scalogram, defined for the CWT, actually represents a local time-frequency energy density of a signal $f$. The energy of a signal $f$ can be represented by the integral of its square or its instantaneous power with respect to time. This energy, using the CWT expansion of $f$ and assuming that we represent $f$ by a finite number of its WT values in a bounded timescale domain (or a time-frequency rectangle related to it through the mother wavelet used) containing most of the signal energy, can be expressed as follows:

$$
\int f^{2}(t) d t=\int\left[\sum_{1 \leq j \leq J} \sum_{n} W f\left(s_{j}, b_{n}\right) \psi_{j n}(t)\right]^{2} d t .
$$

Applying to (9) the orthonormality property of the wavelet basis, we obtain

$$
\begin{aligned}
\int f^{2}(t) & =\sum_{1 \leq j \leq J} \sum_{n} W f\left(s_{j}, b_{n}\right)^{2} \int \psi_{j n}(t)^{2} d t \\
& =\sum_{1 \leq j \leq J} \sum_{n} W f\left(s_{j}, b_{n}\right)^{2} .
\end{aligned}
$$

This can be represented as

$$
\begin{aligned}
\int f^{2}(t) d t=\sum_{n} W f\left(s_{1}, b_{n}\right)^{2} & +\sum_{n} W f\left(s_{2}, b_{n}\right)^{2} \\
& +\cdots+\sum_{n} W f\left(s_{J}, b_{n}\right)^{2}
\end{aligned}
$$


where the first sum is the signal energy in the highest frequency subband (corresponding to the lowest scaling level 1) and the last sum represents the signal energy in the lowest frequency subband (corresponding to the highest scaling level of interest $J$ ). The sequence of the squares of wavelet coefficients $W f\left(s_{j}, b_{n}\right)^{2}$, involved in a particular sum (corresponding to a fixed value of $j$ ), represents the evolution in time of the energy density of $f(t)$ in a bandwidth corresponding to the scaling level $j$. Since (11) is the expression for the signal energy in the time interval of interest with sampling points $n$, the squares of wavelet coefficients $W f\left(s_{j}, b_{n}\right)^{2}$ represent the local energy density of a signal in its time-frequency domain or its scalogram.

Let us assume that we have a single frequency signal $f_{i}(t)$ at the input of a linear system. The energy of such signal will appear concentrated in a certain domain $D_{1}$ in the time-frequency plane. This representation is made through the scalogram or time-frequency energy density of the signal and is shown in Fig. 2.

Outside domain $D_{1}$ or in domain $D_{2}$, the energy of the input signal is almost negligible. If the behavior of the linear system is completely ideal, the energy of the ideal output signal $f_{i o}(t)$ will also be contained within the same timefrequency domain $D_{1}$. However, if some disturbances or nonlinear effects appear in the output, resulting in the actual output signal $f_{a o}(t)$, the timefrequency analysis of the output signal will show essential amounts of energy in domain $D_{2}$. In other words, the energy corresponding to the linear behavior will be always inside domain $D_{1}$, while the energy in domain $D_{2}$ is associated with EMI and nonlinear processes.

According to (10), we can define the domain $D_{1}$, containing $95 \%$ of the input signal energy, as follows:

$$
D_{1}: \sum_{(j, n) \in D_{1}} W f_{i}\left(s_{j}, b_{n}\right)^{2}=0.95 \sum_{j, n} W f_{i}\left(s_{j}, b_{n}\right)^{2} .
$$

We propose to evaluate EMI influence and/or nonlinear effects considering the energy contained in domain $D_{2}$ using the following signal integrity ratio (SIR):

$$
\operatorname{SIR}\left(b_{n}\right)=20 \log _{10}\left(\frac{\sum_{j \in D_{2}} W f_{a o}\left(s_{j}, b_{n}\right)^{2}}{\sum_{j \in D_{2}} W f_{i o}\left(s_{j}, b_{n}\right)^{2}}\right)
$$

where $b_{n}$ is the time shift of the analyzing wavelet and actually represents time. At any time instant $t$, corresponding to a particular $b_{n}$, the SIR takes a value of 0 $\mathrm{dB}$ when the energy of the actual output signal $f_{a o}(t)$ (due to nonlinear phenomena represented by the energy appearing in domain $D_{2}$ ) is identical to that of the ideal one $f_{i o}(t)$. In other words, SIR will be particularly sensitive to the energy pockets appearing in domain $D_{2}$ and not to the energy changes that could appear inside $D_{1}$. In addition, we define the following cumulative energy ratio (CER): 
$\operatorname{CER}\left(b_{n}\right)=20 \log _{10}\left(\frac{\sum_{j \in D_{1} \cup D_{2}} W f_{a o}\left(s_{j}, b_{n}\right)^{2}}{\sum_{j \in D_{1} \cup D_{2}} W f_{i o}\left(s_{j}, b_{n}\right)^{2}}\right)$

that represents the total energy ratio between the actual and the ideal output. At any time instant $t$, corresponding to a particular $b_{n}$, the CER takes a value of 0 $\mathrm{dB}$ when the total energy of the actual output signal $f_{\text {ao }}(t)$, involving both linear and nonlinear effects, is identical to that of the ideal one $f_{i o}(t)$. In other words, CER somehow compares the total energy content of both signals.

Although this methodology holds for general application, in this paper, we deal with audio signals. For this reason, we adopt as an input a chirp signal, whose frequency sweeps linearly with time from $100 \mathrm{~Hz}$ up to $20 \mathrm{kHz}$ in $10 \mathrm{~ms}$. In order to better illustrate these ideas, we will consider simulated examples, in which we assume that this signal is an ideal output. The domains $D_{1}$ and $D_{2}$, determined for such signal according to the definition given in (12), appear in Fig. 3.

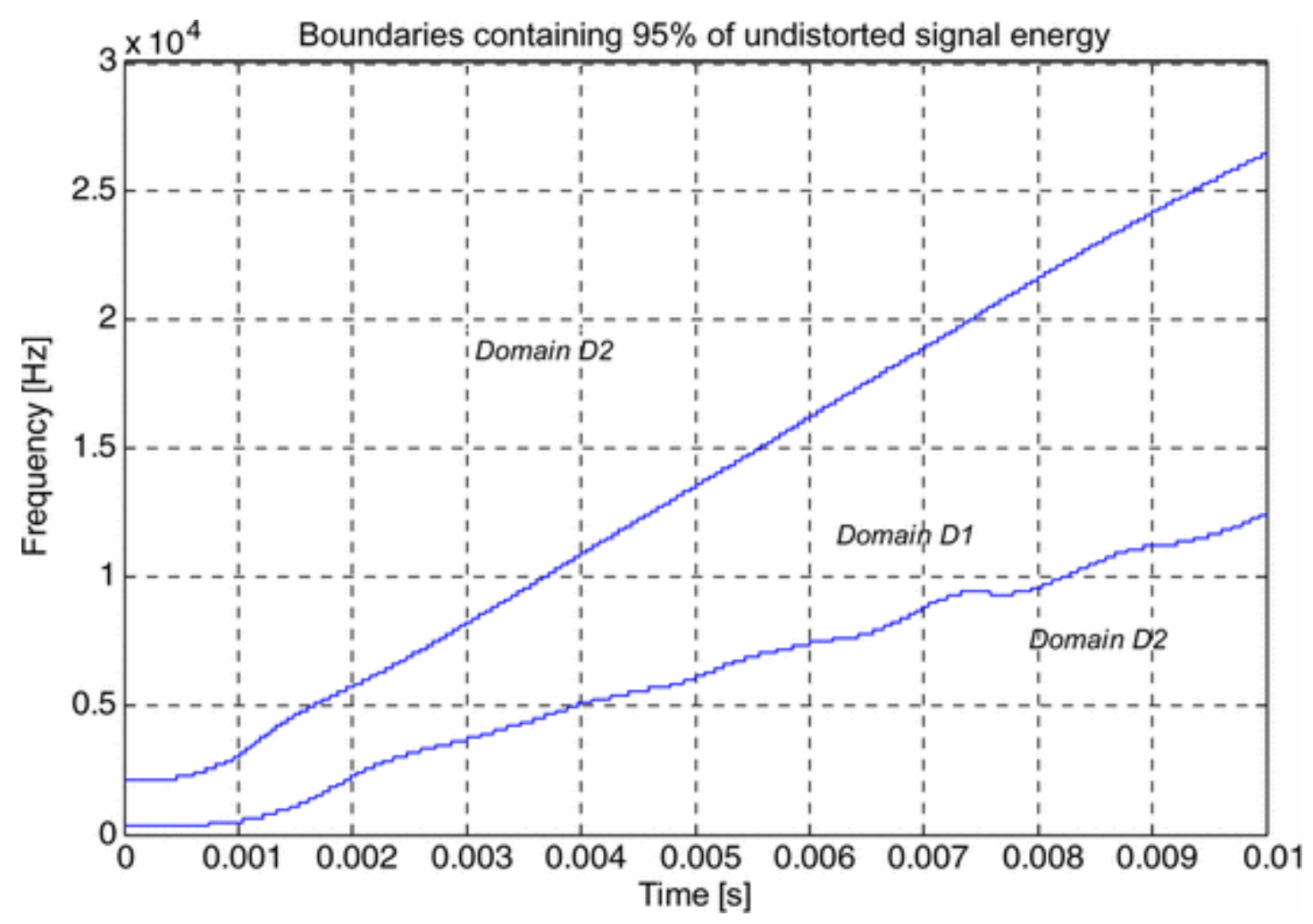

Fig. 3 Domains $D_{1}$ and $D_{2}$ for the ideal output chirp signal.

\section{A) Simulated Example 1}

Consider a chirp signal with three types of disturbances: step change of gain, saturation, and repetitive impulsive noise, known as electric fast transient (EFT). These disturbances may represent a wide variety of conditions. For instance, 
gain changes or saturation effects can appear as a consequence of the nonlinear behavior of a system. On the other hand, the EFT is a very common disturbance present in electronic systems as a consequence of EMI. Fig. 4 shows an increasing frequency chirp signal with visible influence of the step change of gain and saturation. The undistorted signal consists of a chirp signal with constant amplitude that is equal to $1 \mathrm{~V}$. The scalogram of the distorted output appears in Fig. 5.

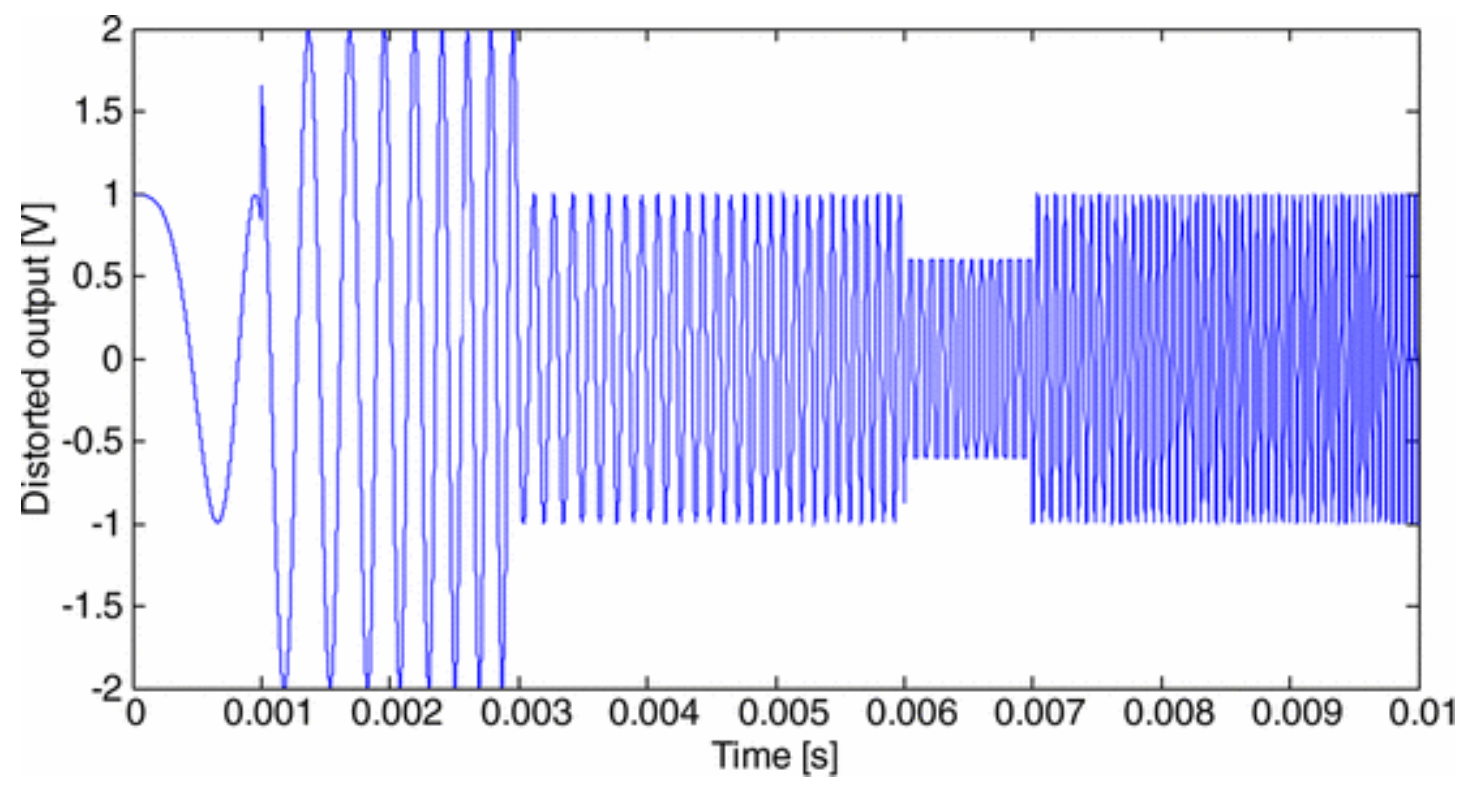

Fig. 4 Distorted increasing frequency chirp with step change of gain and saturation.

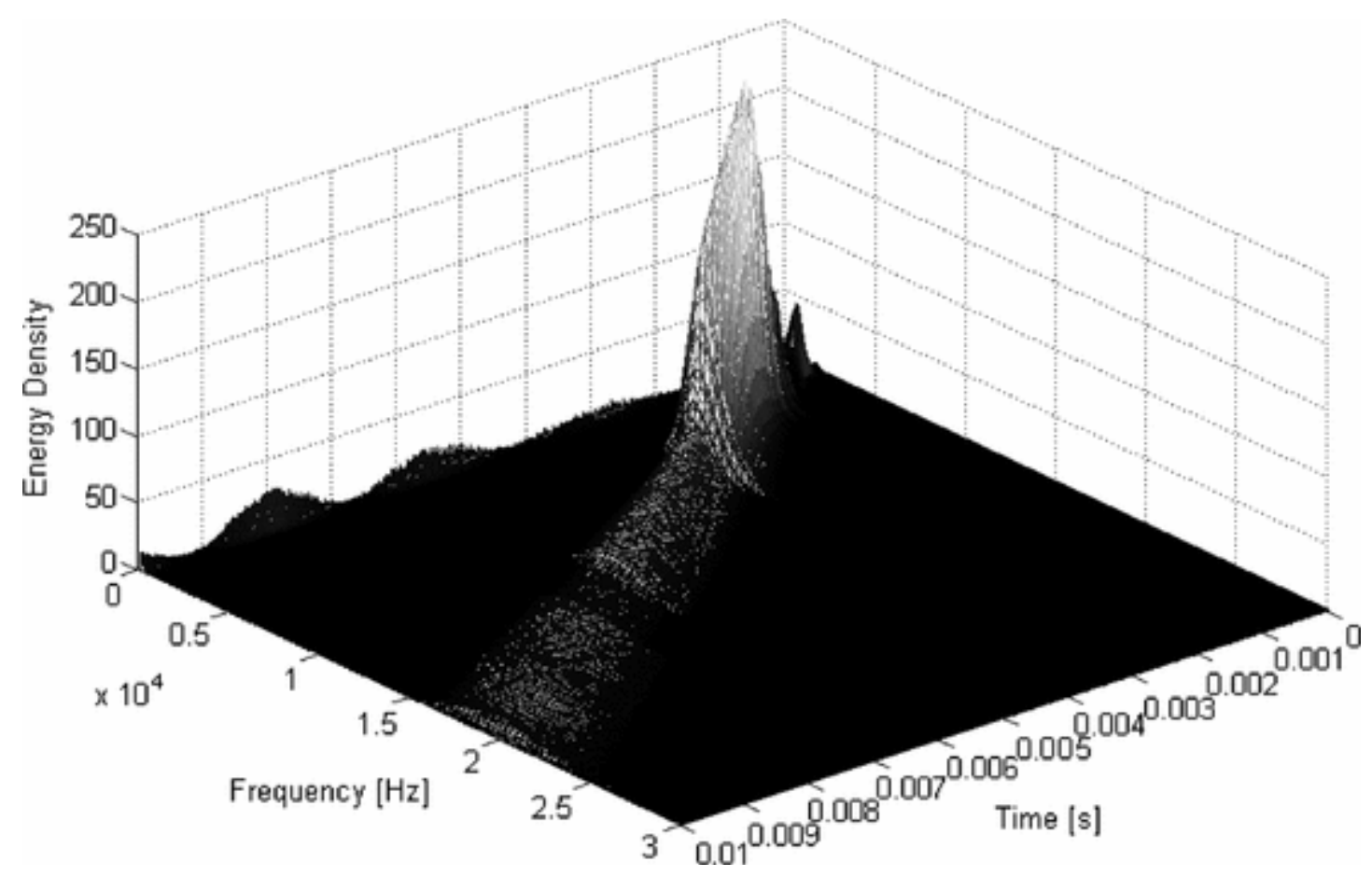

Fig. 5. Scalogram of the distorted output signal. 
Fig. 5 clearly shows the increase in the signal energy density due to gain increase in the interval between 1 and $3 \mathrm{~ms}$ and the drop of energy density due to the saturation effect that occurs in the interval between 6 and $7 \mathrm{~ms}$. In Fig. 6 , the $\operatorname{SIR}(t)_{\text {and }} \operatorname{CER}(t)$ for Simulated Example 1 appear.
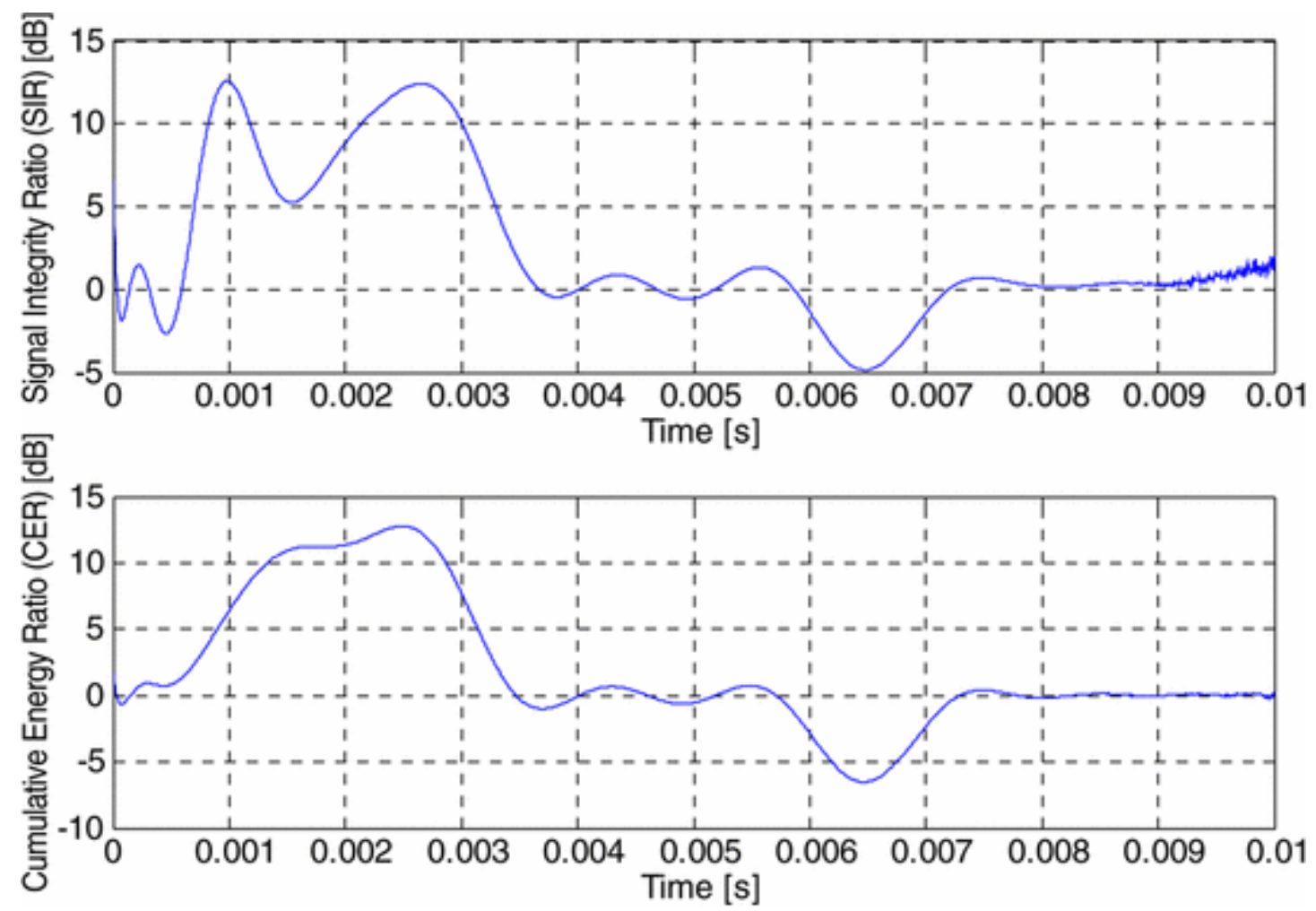

Fig. 6. (Upper panel) SIRand (lower panel) CERas functions of time for Simulated Example 1.

We can see that, due to the increase of gain in the interval between 1 and $3 \mathrm{~ms}$, the value of SIR is $12 \mathrm{~dB}$, corresponding to doubling the amplitude of the signal. On the other hand, the saturation at an amplitude level of 0.6 in the interval between 6 and $7 \mathrm{~ms}$ results in the value of SIR of $5 \mathrm{~dB}$.

\section{A) Simulated Example 2}

Consider a chirp signal with added noise consisting of $15-\mathrm{kHz}$ exponentially damped ringing, shown in Fig. 7. The scalogram of the distorted signal is not shown, but it exhibits an increase in signal energy over the time interval when the disturbance is present. It can also be observed that the maximum of distortion energy appears at $15 \mathrm{kHz}$, i.e., at the frequency of the ringing disturbance. Fig. 8 shows its $\operatorname{SIR}(t)$ and $\operatorname{CER}(t)$. 

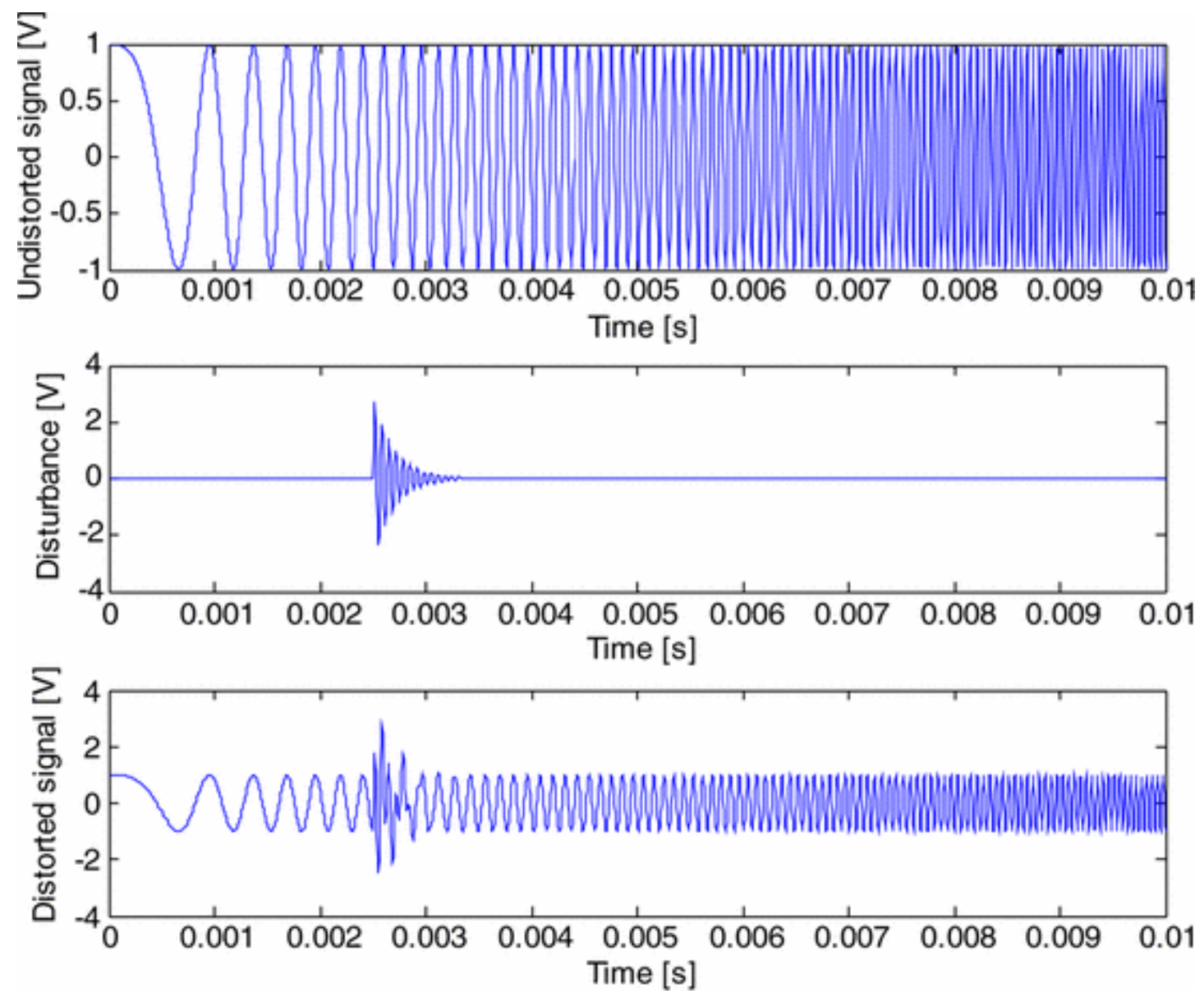

Fig. 7. Chirp signal with exponentially damped ringing disturbance. 

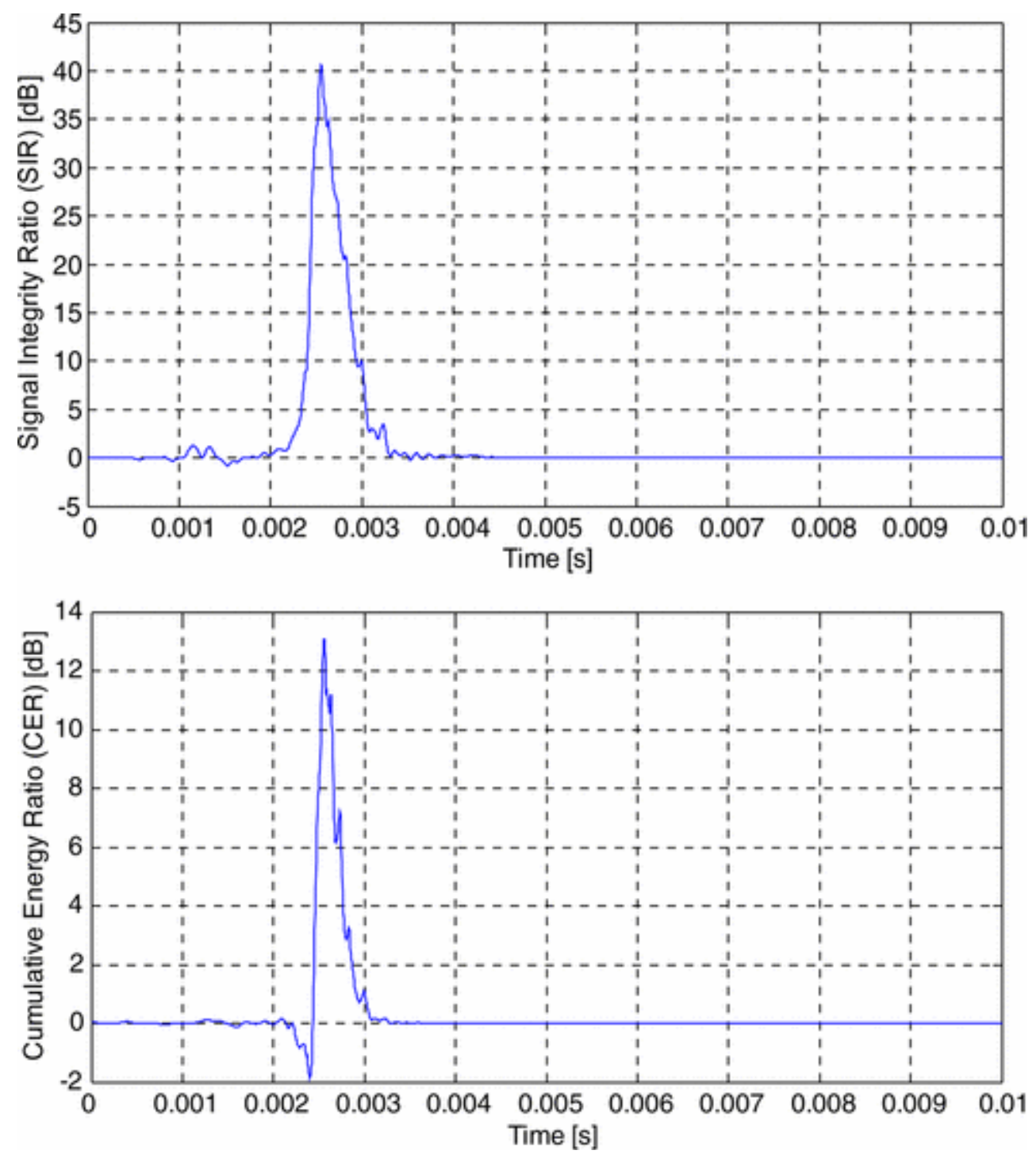

Fig. 8. (Upper panel) $\operatorname{SIR}(t)$ and (lower panel) $\mathrm{CER}(t)$ as functions of time for Simulated Example 2.

From the results of these examples, it is interesting to point out some facts. It is clear that the waveforms of both $\operatorname{SIR}(t)$ and $\operatorname{CER}(t)$ are similar. However, $\operatorname{SIR}(t)$ assumes a much higher value than $\mathrm{CER}(t)$ in Simulated Example 2. The explanation of this fact follows directly from the definitions of these figures of merit.

A much higher value of SIR than CER shows that a significant amount of energy is present in domain $D_{2}$. For instance, in Simulated Example 2, the amplitude of the damped oscillation is $3 \mathrm{~V}$ in comparison to $1 \mathrm{~V}$ of the undistorted output. This leads us to the maximum values of 40 and $13 \mathrm{~dB}$ for $\operatorname{SIR}(t)$ and $\operatorname{CER}(t)$, respectively, while in Simulated Example 1, both functions take almost the same maximum value. 
SECTION III

\section{SIGNAL INTEGRITY ANALYSIS APPLIED TO THE EVALUATION OF AN AUDIO AMPLIFIER}

In order to validate the proposed methodology of the wavelet-based signal integrity analysis, introduced in Section II, we have applied it to the performance evaluation of a real audio amplifier, as reported in Experiment 1.

\section{A) Experiment 1}

We evaluate the performance of an audio amplifier with the saturation effect shown in Fig. 9.

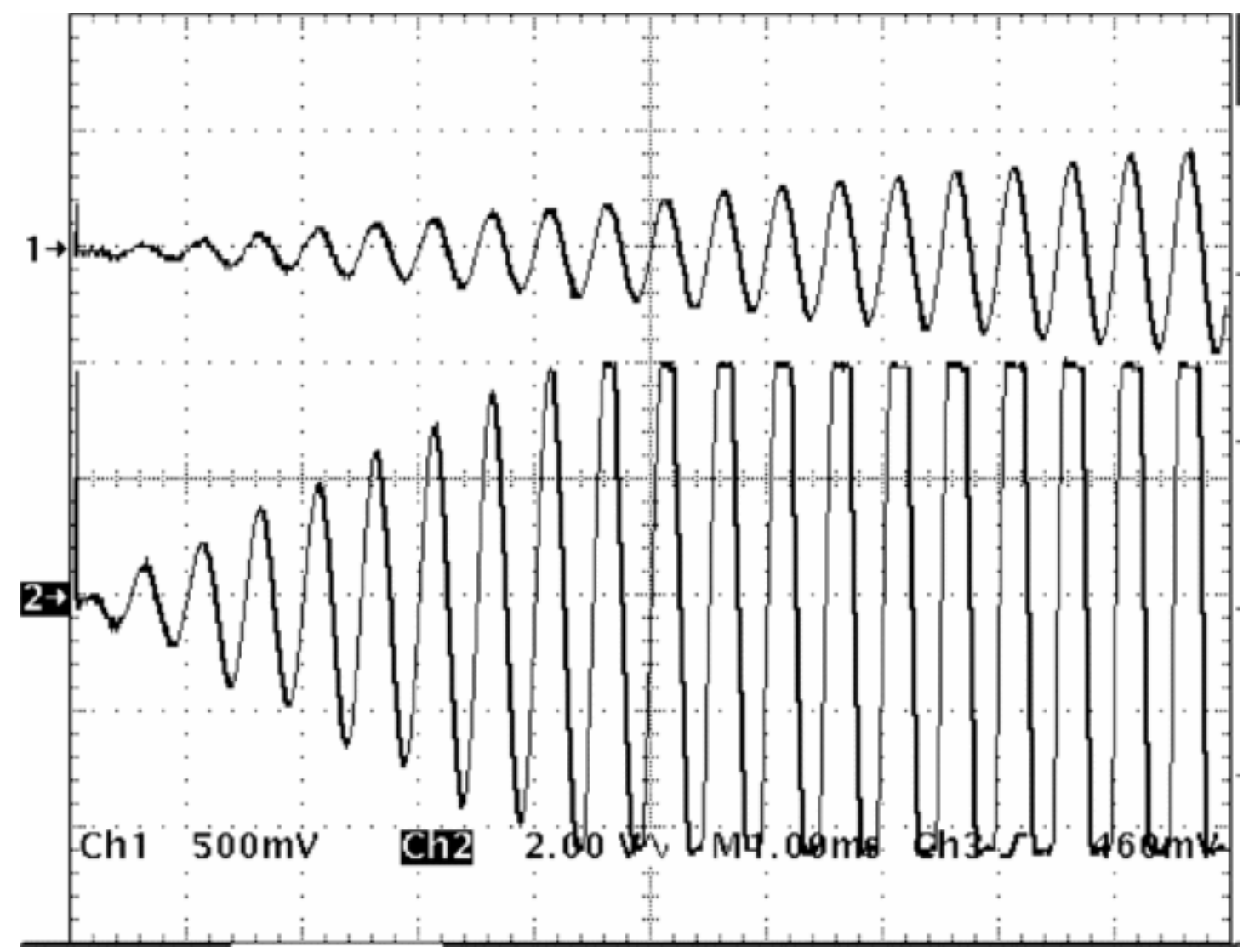

Fig. 9. (Upper trace) Input and (lower trace) output with saturation.

The input signal has a constant frequency of $2 \mathrm{kHz}$, and its amplitude increases linearly with time. The output signal behaves in the same way, until saturation is reached. The amplifier gain remains constant and equals 20.

Fig. 10 shows the scalograms of the input voltage (upper plot) and the output voltage (lower plot). The frequency content of the input signal remains constant, and the energy density of the signal increases with time. In this figure, it can be also seen that the most important part of the output signal energy remains around $2 \mathrm{kHz}$. However, a significant amount of energy appears centered 
around $6 \mathrm{kHz}$ when saturation occurs. This is the energy corresponding to the third-order harmonic that appears as a consequence of the saturation effect.
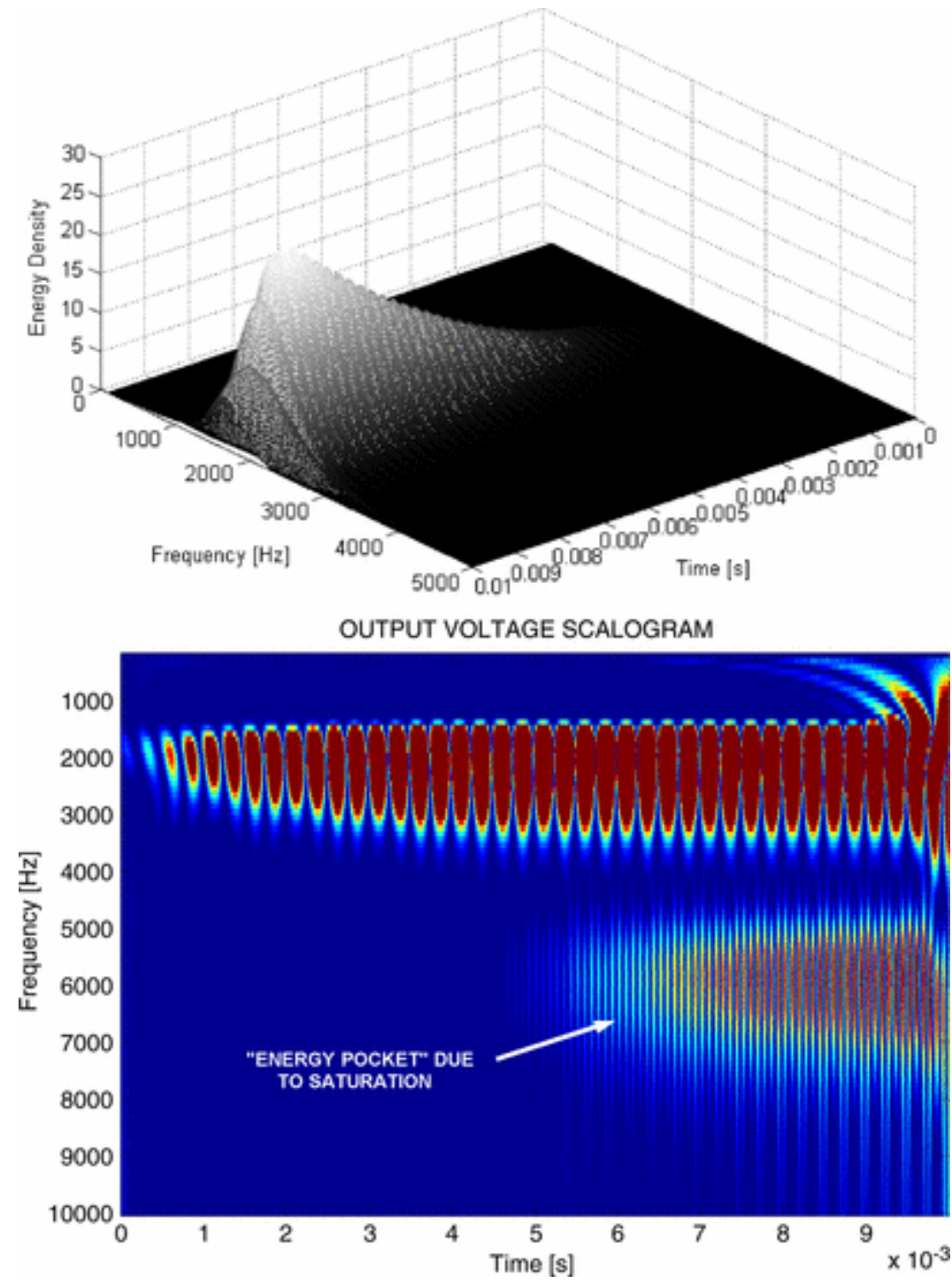

Fig. 10. Scalogram of (upper plot) the input signal and (lower plot) the output signal with saturation.

This energy represents the main contribution to the full amount of energy contained in domain $D_{2}$. The discussed effect is also reflected by the SIR, which appears in Fig. 11. The saturation effect is reflected by the CER as well, which is shown in Fig. 12. When there is no saturation effect, i.e., for time interval below $5 \mathrm{~ms}$ in Fig. 12, the value of CER is $0 \mathrm{~dB}$. However, when the saturation effect is present in the output (for times above $5 \mathrm{~ms}$ ), the value of CER drops. This happens because saturation entails the spread of energy in several harmonics, originally ideally concentrated around $2 \mathrm{kHz}$. It should be considered that wavelet analysis is carried out within a finite frequency range (up to $20 \mathrm{kHz}$ in this case) and many of these harmonics are not accounted for because they are out of this range. In addition, it should be noticed that the energy content at the fundamental frequency ( $2 \mathrm{kHz}$ in this case) is much bigger in the case of the ideal output than for the saturated output. 


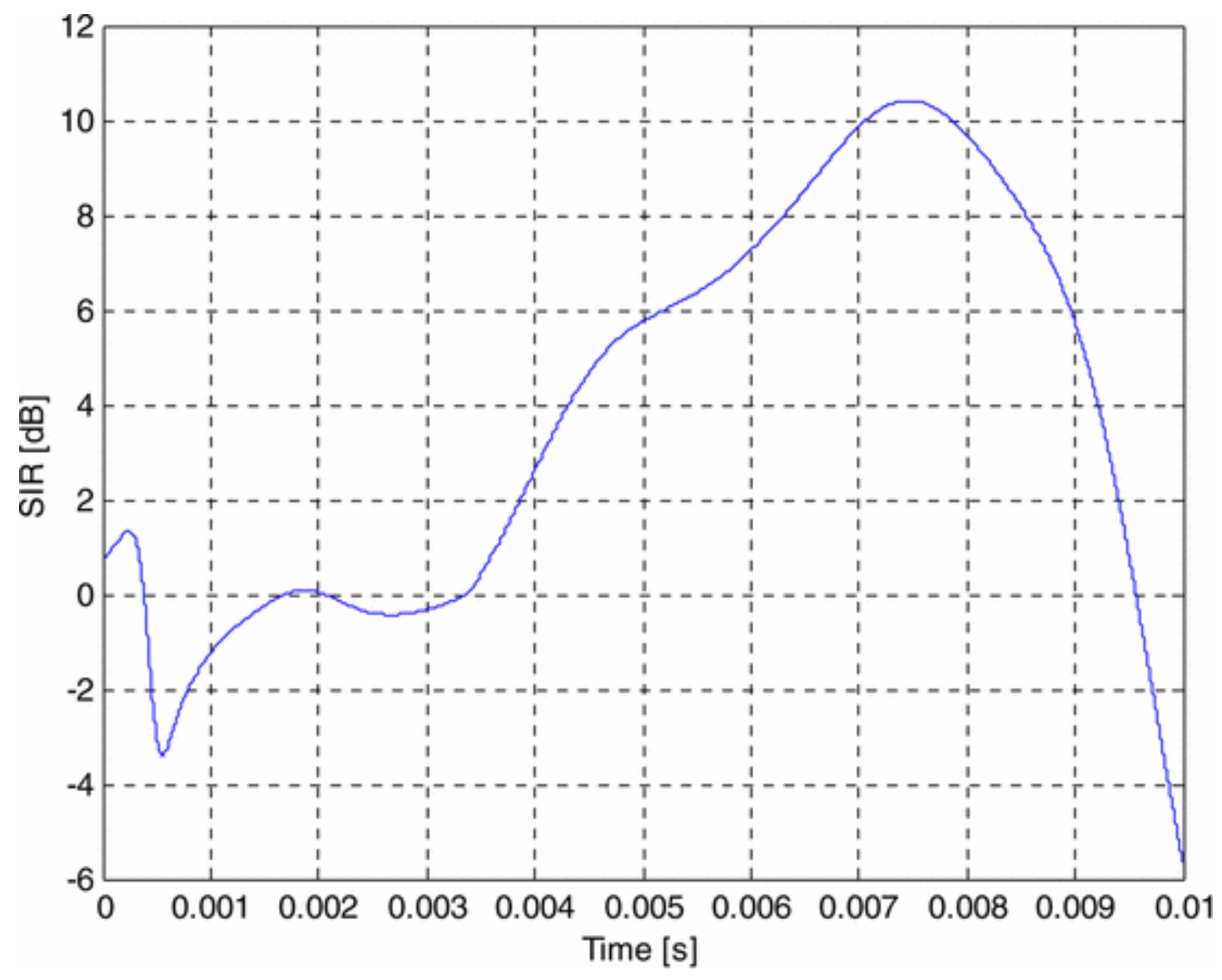

Fig. 11. SIR for the output signal with saturation.

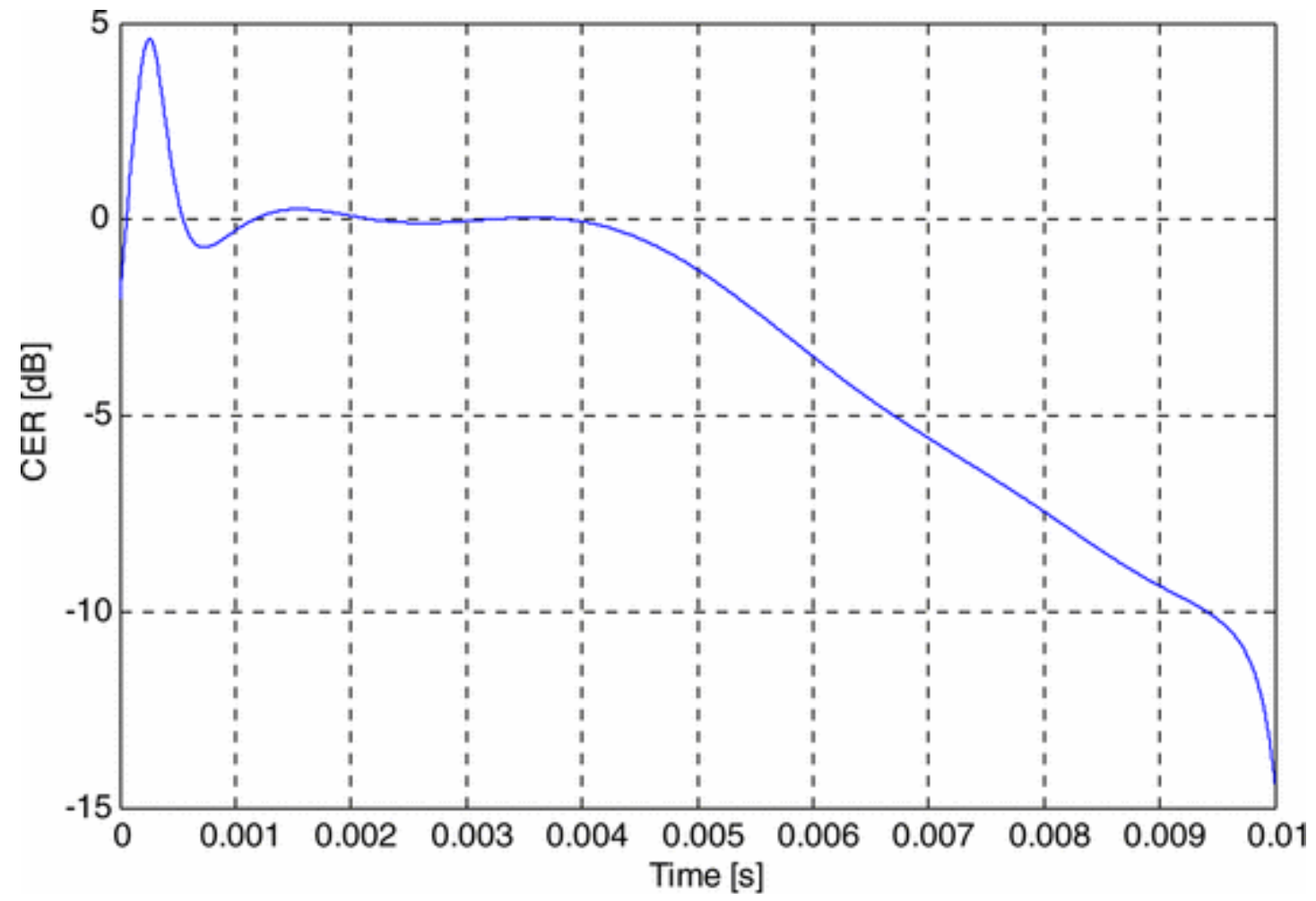

Fig. 12. CER for output signal with saturation. 


\section{A) Experiment 2}

In this experiment, we analyze a change in the time-frequency energy distribution of an audio amplifier output signal induced by an amplitudemodulated noise added to the input (a chirp signal sweeping from $20 \mathrm{~Hz}$ up to $20 \mathrm{kHz}$ in $10 \mathrm{~ms}$ ). In the upper panel of Fig. 13, one can see the undistorted output, while in the lower panel appears the output distorted with an amplitudemodulated noise added to the same input. Looking at this distorted signal, one cannot tell anything about the kind of noise involved.
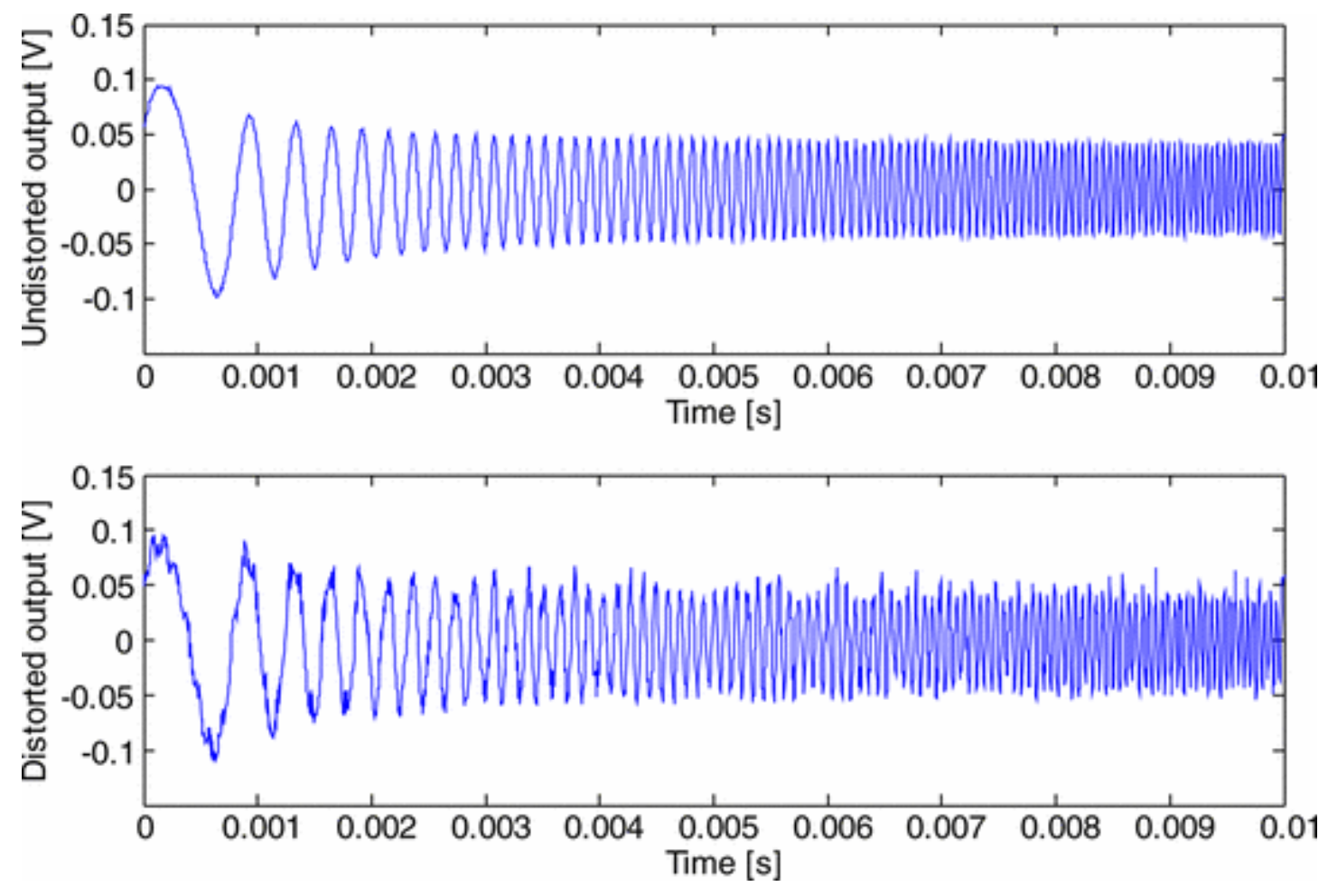

Fig. 13 Output effect of noise added to the input chirp signal.

We obtained scalograms of both output signals. They are shown in Fig. 14 for undistorted output and in Fig. 15 for distorted output. In Fig. 14, the energy of the signal is clearly concentrated inside the domain $D_{1}$ (typical for the chirp signal) with the borders similar to those shown in Fig. 3. 


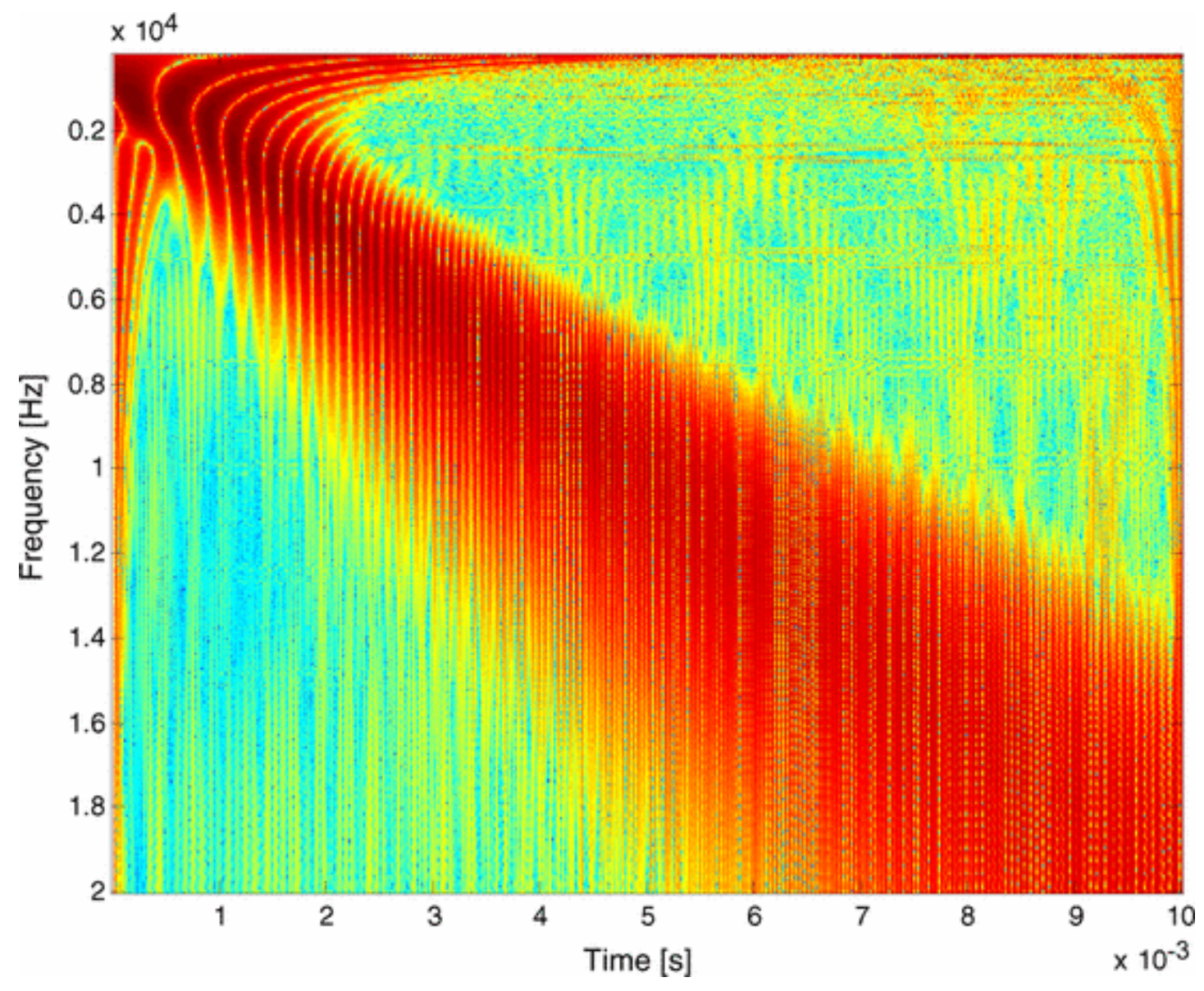

Fig. 14. Wavelet scalogram of response to the undistorted input chirp signal.

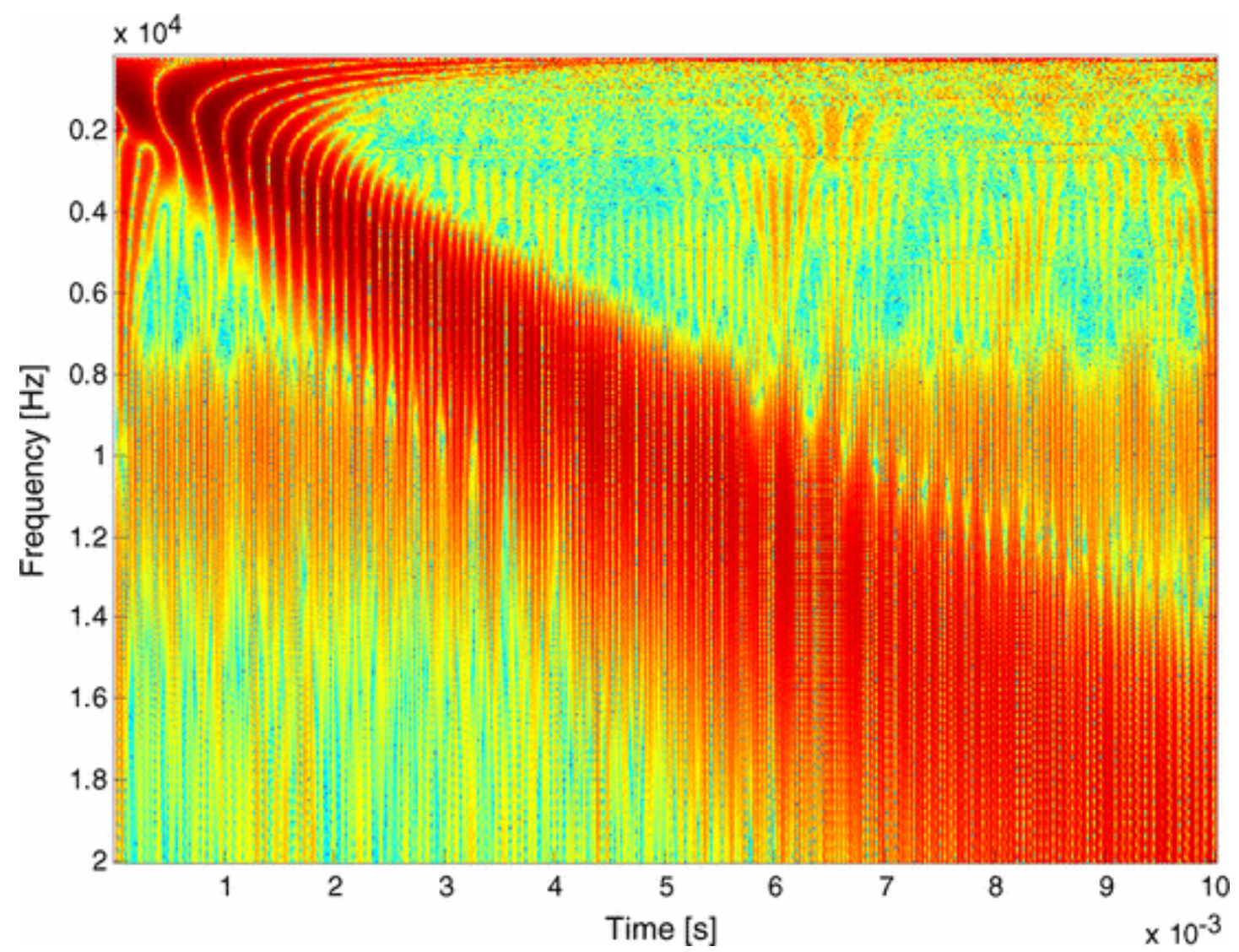


Fig. 15. Wavelet scalogram of the response to the distorted input chirp signal.

In Fig. 15, the scalogram of the distorted output appears. The additional energy concentration at a fixed frequency of $10 \mathrm{kHz}$ can be clearly observed. This is the carrier frequency of the amplitude-modulated noise. In order to clearly show this fact, we present for both signals (in Fig. 16) the energy density at $10 \mathrm{kHz}$ over their duration.
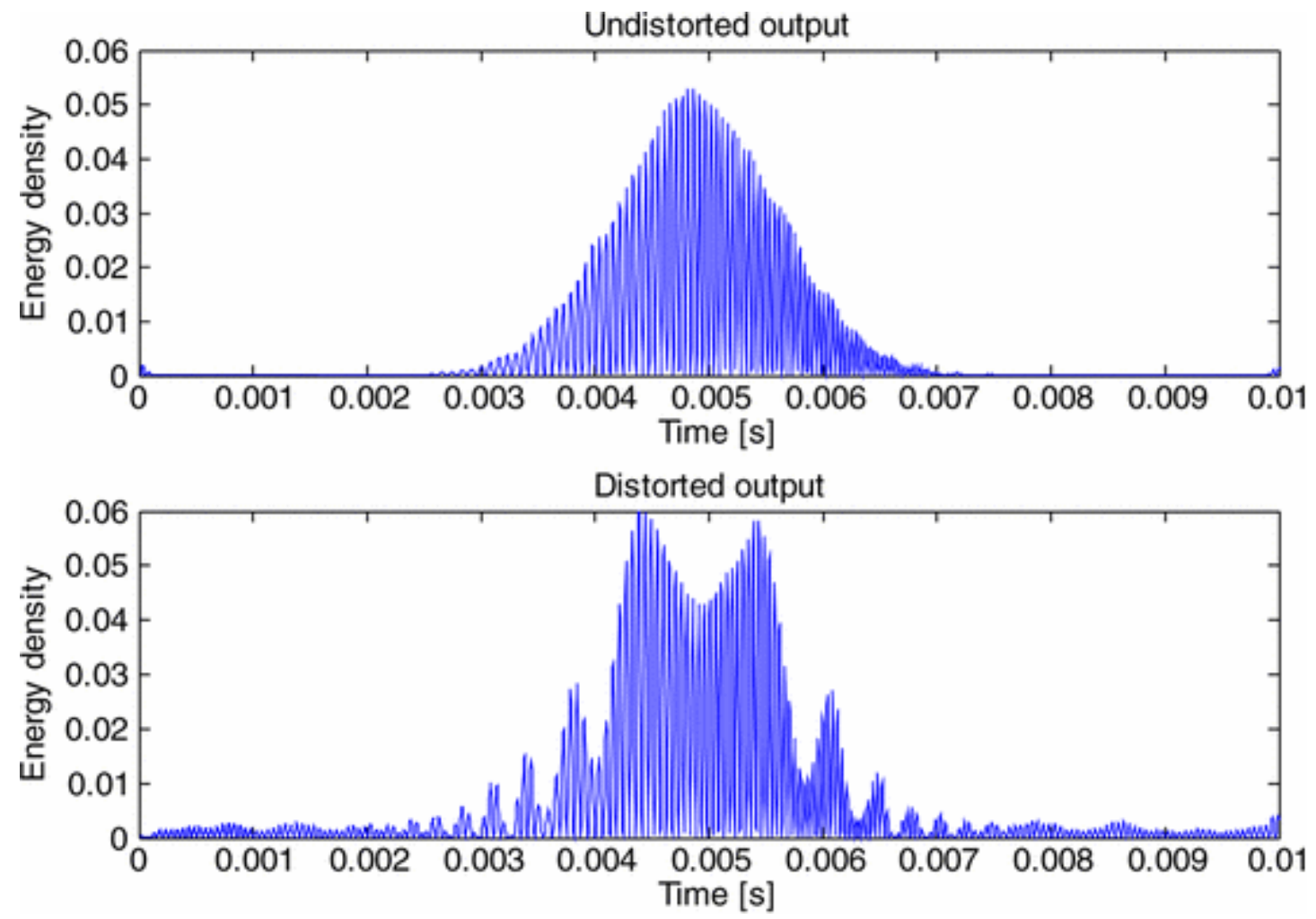

Fig. 16. Output energy density at $10 \mathrm{kHz}$ in (upper panel) undistorted signal and (lower panel) distorted signal.

The upper panel characterizes the undistorted signal and shows a peak of energy slightly below $5 \mathrm{~ms}$, i.e., approximately in the middle of the frequency sweep of the chirp signal. At this time instant, the chirp signal reaches this frequency value. The energy content at $10 \mathrm{kHz}$ for all the other values of time (below $3 \mathrm{~ms}$ and above $7 \mathrm{~ms}$ ) is almost negligible.

In the lower panel, the energy content at $10 \mathrm{kHz}$ for distorted signal appears. There are several facts to underline. First of all, some energy content is present during the whole duration of the signal (10-ms interval). This energy corresponds to the amplitude-modulated noise carrier of $10 \mathrm{kHz}$ present in the output (notice the energy below $3 \mathrm{~ms}$ and above $7 \mathrm{~ms}$ ). Moreover, some peaks of energy appear with a period of $0.5 \mathrm{~ms}$ approximately. They are related to the modulation frequency of the amplitude-modulated noise. Finally, a large concentration of energy appears around $5 \mathrm{~ms}$ due to the original chirp signal, but its magnitude is influenced by the superimposed energy of the noise. 


\section{SECTION IV}

\section{CONCLUSION}

In this paper, we have developed a general procedure along with new tools for the evaluation of influence of EMI and nonlinear processes on system performance as represented by signal integrity. We propose to evaluate signal integrity using time-frequency energy density represented by the wavelet scalogram. Using this concept, we can determine the time-frequency domain in which most of the input signal energy is contained. As a result of nonlinear and/or EMI effects, substantial energy of the output signal may appear outside this time-frequency domain. In fact, our analysis considers the difference in energy distribution in the time-frequency domain between distorted and undistorted signals. The performance indexes SIR and CER represent the extent to which the integrity of a signal is diminished by EMI effects and/or nonlinear processes. Simulated examples are used to illustrate signal integrity analysis performed with these tools, followed by a demonstration of their usefulness using experimental data of a real audio amplifier.

\section{APPENDIX}

In the presented application, the WT scalogram of a signal represents its energy density localized in frequency and time that can best be achieved by the use of mother wavelet that is a windowed sinusoid. What makes the Morlet WT (MWT) best suited for our application is that the width of its Gaussian window is coupled to the center frequency $\omega_{0}$. This reduces the window width at higher frequencies that ensures that the number of cycles under the Gaussian remains the same for all frequencies. The mother wavelet at frequency $\omega_{0}$

$\psi(t)=e^{\frac{-t^{2}}{2}} \cos \omega_{0} t$

and the wavelet at frequency $\omega \psi(t, \omega)=e^{\frac{-t^{2}}{2 \sigma_{t}^{2}}} \cos \omega t$

where the standard deviation $\sigma_{t i s}$ inversely proportional to the frequency win order to retain the wavelet scaling properties. For $n_{0}$ significant wavelet cycles, we have

$$
\sigma_{t}=\frac{n_{0}}{\omega} .
$$

It is easy to show that, choosing a Gaussian window for the short-time Fourier transform (STFT), it can be made equivalent to the MWT for a particular frequency. Similar equivalence can be found applying the Hilbert transform to bandpass-filtered data (BP/HT). However, the STFT and BP/HT use the same window width at each frequency, whereas for the MWT, the width is a function of frequency. 


\section{ACKNOWLEDGMENT}

J. T. Bialasiewicz would like to thank the Spanish Ministry of Education, Culture, and Sports for the support given for his sabbatical under the Mobility Program for Foreign Professors.

\section{Footnotes}

This work was supported by Spanish Ministry of Science and Innovation under Project TEC2011-25076/MIC.

J. T. Bialasiewicz is with the Department of Electrical Engineering, University of Colorado Denver, Denver, CO 80217 USA, and also with the Polish-Japanese Institute of Information Technology, 02-008 Warsaw, Poland (e-mail: Jan.Bialasiewicz@ucdenver.edu).

D. González, J. Balcells, and J. Gago are with the Electronics Engineering Department, Universitat Politècnica de Catalunya, 08034 Barcelona, Spain (email: david.gonzalez@upc.edu; josep.balcells@upc.edu; javier.gago@upc.edu).

Color versions of one or more of the figures in this paper are available online at http://ieeexplore.ieee.org.

\section{References}

1. G. Antonini and A. Orlandi, "Wavelet packet-based EMI signal processing and source identification"IEEE Trans. Electromagn. Compat., vol. EMC-43, no. 2, pp. 140-148, 2001

2. D. González, J. T. Bialasiewicz, J. Balcells and J. Gago. "Wavelet-based performance evaluation of power converters operating with modulated switching frequency". IEEE Trans. Ind. Electron., vol. 55, no. 8, pp. 3167-3176, 2008

3. G. Antonini and A. Orlandi. "Lightning-induced effects on lossy MTL terminated on arbitrary loads: A wavelet approach" IEEE Trans. Electromagn. Compat., vol. 42, no. 2, pp. 181-189, 2000

4. C. Buccella and A. Orlandi. "Diagnosing transmission line termination faults by means of wavelet based crosstalk signature recognition". IEEE Trans. Compon., Packag., Manuf. Technol., vol. 23, no. 1, pp. 165-170, 2000

5. P. Clarkson and P. S. Wright. "A wavelet-based method of measuring fluctuating harmonics for determining the filter time constant of IEC standard harmonic analyzers". IEEE Trans. Instrum. Meas., vol. 54, no. 2, pp. 488-491, 2005

6. N. D. Kelley, R. M. Osgood, J. T. Bialasiewicz and A. Jakubowski. "Using wavelet analysis to assess turbulence/rotor interactions". Wind Energy, vol. 3, no. 3, pp. 121-134, 2000 
7. N. D. Kelley, B. J. Jonkman, J. T. Bialasiewicz, G. N. Scott and L. S. Redmond. "The impact of coherent turbulence on wind turbine aeroelastic response and its simulation". Proc. AWEA Windpower, 2005

8. C.-H. Lu. "Design and application of stable predictive controller using recurrent wavelet neural networks". IEEE Trans. Ind. Electron., vol. 56, no. 9, pp. 3733-3742, 2009.

9. S. P. Valsan and K. S. Swarup. "High-speed fault classification in power lines: Theory and FPGA-based implementation". IEEE Trans. Ind. Electron., vol. 56, no. 5, pp. 1793-1800, 2009

10. C. Wen, C. Zhu, Y. Ju, Y. Qiu, H. Xu and W. Lu. "Optimal frequency band design scheme of dyadic wavelet processor array using surface acoustic wave devices". IEEE Trans. Ind. Electron., vol. 56, no. 4, pp. 949-955, 2009

11. X. Chen. "Real wavelet transform-based phase information extraction method: Theory and demonstrations". IEEE Trans. Ind. Electron., vol. 56, no. 3, pp. 891-899, 2009

12. M. A. S. K. Khan and M. A. Rahman. "Implementation of a wavelet-based MRPID controller for benchmark thermal system". IEEE Trans. Ind. Electron., vol. 57, no. 12, pp. 4160-4169, 2010.

13. A. Y. Goharrizi and N. Sepehri. "A wavelet-based approach to internal seal damage diagnosis in hydraulic actuators". IEEE Trans. Ind. Electron., vol. 57, no. 5, pp. 1755-1763, 2010.

14. S. A. Saleh, C. R. Moloney and M. A. Rahman. "Analysis and development of wavelet modulation for three-phase voltage-source inverters". IEEE Trans. Ind. Electron., vol. 58, no. 8, pp. 3330-3348, 2011

15. C.-H. Lu. "Wavelet fuzzy neural networks for identification and predictive control of dynamic systems". IEEE Trans. Ind. Electron., vol. 58, no. 7, pp. 3046-3058, 2011

16. J. Pons-Llinares, J. A. Antonino-Daviu, M. Riera-Guasp, M. Pineda-Sanchez and V. Climente-Alarcon. "Induction motor diagnosis based on a transient current analytic wavelet transform via frequency B-splines". IEEE Trans. Ind. Electron., vol. 58, no. 5, pp. 1530-1544, 2011

17. A. Bouzida, O. Touhami, R. Ibtiouen, A. Belouchrani, M. Fadel and A. Rezzoug. "Fault diagnosis in industrial induction machines through a discrete wavelet transform". IEEE Trans. Ind. Electron., vol. 58, no. 9, pp. 4385-4395, 2011

18. S. Mallat. A Wavelet Tour of Signal Processing. 2009, Academic Press

19. J. T. Bialasiewicz. Falki i Aproksymacje. 2004, WNT 
20. D. González, J. Balcells and J. T. Bialasiewicz. "Exploration of application of continuous wavelet transform to power quality analysis". Proc. ISIE, pp. 22422246, 2008

21. S. Grivet-Talocia and F. G. Canavero. "Wavelet-based high-order adaptive modeling of lossy interconnects". IEEE Trans. Electromagn. Compat., vol. 43, no. 4, pp. 471-484, 2001

22. S. Barmada, A. Musolino and M. Raugi. "Wavelet-based time-domain solution of multiconductor transmission lines with skin proximity effect". IEEE Trans. Electromagn. Compat., vol. 47, no. 4, pp. 774-780, 2005

23. S. Barmada, A. Musolino, M. Raugi, R. Rizzo and M. Tucci. "A wavelet based method for the analysis of impulsive noise due to switch commutations in power line communication (PLC) systems". IEEE Trans. Smart Grid, vol. 2, no. 1, pp. 92-101, 2011

24. L. Coppola, S. Buso, Q. Liu, D. Boroyevich and A. Bell. "Application of Fourier and wavelet transforms to the identification of EMI noise sources in SMPSs". Proc. IEEE Int. Symp. EMC, pp. 584-589, 2005

25. S. Molla and B. Torresani. "Determining local transientness of audio signals". IEEE Signal Process. Lett., vol. 11, no. 7, pp. 625-628, 2004

26. J. S. Colburn, H. J. Song, H. P. Hsu and R. Wiese. "Assessment of automobile radio system performance in noisy EM environments". Proc. IEEE Int. Symp. EMC, pp. 24-28, 2005 